VOL. 70 (2004) [391-395]

\title{
ON THE ORDERS OF GENERATORS OF CAPABLE $p$-GROUPS
}

\author{
ARTURo MAGIDIN
}

\begin{abstract}
A group is called capable if it is a central factor group. For each prime $p$ and positive integer $c$, we prove the existence of a capable p-group of class $c$ minimally generated by an element of order $p$ and an element of order $p^{1+\lfloor c-1 / p-1\rfloor}$. This is best possible.
\end{abstract}

\section{INTRODUCTION}

Recall that a group $G$ is said to be capable if and only if $G$ is isomorphic to $K / Z(K)$ for some group $K$, where $Z(K)$ is the centre of $K$. There are groups which are not capable (nontrivial cyclic groups being a well-known example), so capability places restrictions on the structure of a group; see for example $[3,4]$. As noted by Hall in his landmark paper on the classification of $p$-groups ([2]), the question of which $p$-groups are capable is interesting and plays an important role in their classification.

Hall observed that if $G$ is a capable $p$-group of class $c$, with $c<p$, and $\left\{x_{1}, \ldots, x_{n}\right\}$ is a minimal set of generators with $o\left(x_{1}\right) \leqslant o\left(x_{2}\right) \leqslant \cdots \leqslant o\left(x_{n}\right)$ (where $o(g)$ denotes the order of the element $g$ ), then $n>1$ and $o\left(x_{n-1}\right)=o\left(x_{n}\right)$.

In [5] we used commutator calculus to derive a similar necessary condition after dropping the hypothesis $c<p$ : if $G$ is a capable $p$-group of class $c>0$, minimally generated by $\left\{x_{1}, \ldots, x_{n}\right\}$, where $o\left(x_{1}\right) \leqslant \cdots \leqslant o\left(x_{n}\right)$, then we must have $n>1$ and letting $o\left(x_{n-1}\right)=p^{a}$ and $o\left(x_{n}\right)=p^{b}$, then $a$ and $b$ must satisfy

$$
b \leqslant a+\left\lfloor\frac{c-1}{p-1}\right\rfloor,
$$

where $\lfloor x\rfloor$ is the greatest integer less than or equal to $x$ ([5, Theorem 3.19]). The dihedral group of order $2^{c+1}$ shows that (1.1) is best possible when $p=2$. The purpose of this note is to show that the inequality is best possible for all primes $p$, thus answering in the affirmative [5, Question 3.22].

Notation will be standard; all groups will be written multiplicatively, and we shall denote the identity by $e$. We use the convention that the commutator of two elements $x$ and $y$ is $[x, y]=x^{-1} y^{-1} x y$. The lower central series of $G$ is defined recursively by letting

Received 13th May, 2004

I thank Professor Avinoam Mann for bringing Easterfield's paper [1] to my attention. It proved invaluable in suggesting the construction in Section 2.

Copyright Clearance Centre, Inc. Serial-fee code: 0004-9727/04 \$A2.00+0.00. 
$G_{1}=G$, and $G_{n+1}=\left[G_{n}, G\right]$. We say $G$ is nilpotent of class (at most) $c$ if and only if $G_{c+1}=\{e\}$. It is well known that if $G$ is of class exactly $c$, then $G_{c} \subset Z(G)$, and $G / Z(G)$ is nilpotent of class exactly $c-1$.

We let $C_{n}$ denote the cyclic group of order $n$, and $\mathbf{Z}$ the infinite cyclic group, both written multiplicatively.

\section{THE CASE $c=1+(r-1)(p-1)$}

The construction in this section is based on the example given by Easterfield in [1, Section 4].

Let $p$ be a prime, $r$ a positive integer. We construct a $p$-group $K$ of class $c+1$ $=2+(r-1)(p-1)$, minimally generated by an element $y$ of order $p$, and an element $x_{0}$ of order $p^{r}$. We shall show that the images of $y$ and $x_{0}$ have the same order in $K / Z(K)$, thus exhibiting a capable group of class $c=1+(r-1)(p-1)$, minimally generated by an element of order $p$ and one of order $p^{1+\lfloor c-1 / p-1\rfloor}$.

Let $H$ be the Abelian group

$$
H=C_{p^{r}} \times C_{p^{r}} \times \underbrace{C_{p^{r-1}} \times \cdots \times C_{p^{r-1}}}_{p-2 \text { factors }} .
$$

Denote the generators of the cyclic factors of $H$ by $x_{0}, x_{1}, \ldots, x_{p-1}$, respectively. If $r=1$, then $x_{2}, \ldots, x_{p-1}$ are trivial. Let $y$ generate a cyclic group of order $p$, and let $y$ act on $H$ by $y^{-1} x_{i} y=x_{i} x_{i+1}$ for $0 \leqslant i \leqslant p-2$ (so $\left[x_{i}, y\right]=x_{i+1}$ ), and

$$
y^{-1} x_{p-1} y=x_{1}^{-\left(\begin{array}{l}
p \\
1
\end{array}\right)} x_{2}^{-\left(\begin{array}{l}
p \\
2
\end{array}\right)} \cdots x_{p-2}^{-\left(\begin{array}{l}
p \\
p-2
\end{array}\right)} x_{p-1}^{1-\left(\begin{array}{c}
p \\
p-1
\end{array}\right)}
$$

as usual, $\left(\begin{array}{l}n \\ k\end{array}\right)$ is the binomial coefficient $n$ choose $k$. Let $K=H \rtimes\langle y\rangle$.

REMARK 2.1. The group constructed by Easterfield is the subgroup of $K$ generated by $y$ and $x_{1}, \ldots, x_{\mathrm{r}}$. We can also realise $K$ as the semidirect product of this subgroup by $\left\langle x_{0}\right\rangle$, letting $x_{0}$ act on $y$ by $x_{0}^{-1} y x_{0}=y x_{1}^{-1}$, and act trivially on the $x_{i}$.

Note that $K$ is metabelian of class exactly $2+(r-1)(p-1)$. To verify the class, 
note that $[K, K]=\left\langle x_{1}, \ldots, x_{p-1}\right\rangle$. We then have:

$$
\begin{aligned}
K_{3} & =\left\langle x_{1}^{p}, x_{2}, \ldots, x_{p-1}\right\rangle ; \\
K_{4} & =\left\langle x_{1}^{p}, x_{2}^{p}, x_{3}, \ldots, x_{p-1}\right\rangle ; \\
& \vdots \\
K_{2+(p-1)} & =\left\langle x_{1}^{p}, x_{2}^{p}, \ldots, x_{p-1}^{p}\right\rangle ; \\
K_{2+(p-1)+1} & =\left\langle x_{1}^{p^{2}}, x_{2}^{p}, \ldots, x_{p-1}^{p}\right\rangle ; \\
& \vdots \\
K_{2+k(p-1)} & =\left\langle x_{1}^{p^{k}}, x_{2}^{p^{k}}, \ldots, x_{p-1}^{p^{k}}\right\rangle ; \\
& \vdots \\
K_{2+(r-1)(p-1)} & =\left\langle x_{1}^{p^{r-1}}, x_{2}^{p^{r-1}}, \ldots, x_{p-1}^{p^{r-1}}\right\rangle=\left\langle x_{1}^{p^{r-1}}\right\rangle .
\end{aligned}
$$

Finally, note that $x_{1}^{p^{r-1}}$ is central: $y^{-1} x_{1}^{p^{r-1}} y=\left(x_{1} x_{2}\right)^{p^{r-1}}=x_{1}^{p^{r-1}}$. Therefore $K$ is of class exactly $2+(r-1)(p-1)$.

The group $G=K / Z(K)$ will therefore be of class $1+(r-1)(p-1)$, minimally generated by $y Z(K)$ and $x_{0} Z(K)$. The order of $y Z(K)$ is of course equal to $p$. As for $x_{0} Z(K)$, note that no nontrivial power of $x_{0}$ is central: if $x_{0}^{k}$ is central, then

$$
x_{0}^{k}=y^{-1} x_{0}^{k} y=\left(y^{-1} x_{0} y\right)^{k}=\left(x_{0} x_{1}\right)^{k}=x_{0}^{k} x_{1}^{k}
$$

therefore $x_{1}^{k}=e$, which implies that $p^{r} \mid k$, so $x_{0}^{k}=e$. Therefore, the order of $x_{0} Z(K)$ is $p^{\tau}$. Thus, $G$ is a capable group of class $c$, with $c=1+(r-1)(p-1)$, minimally generated by an element of order $p$ and an element of order $p^{r}=p^{1+\lfloor c-1 / p-1\rfloor}$.

We note the following fact about $K$, which we shall use in the following section:

LEMMA 2.2. Let $p$ be any prime, and let $r$ be an arbitrary positive integer. There exists a group $K$ of class $2+(r-1)(p-1)$, generated by elements $y$ and $x_{0}$ of orders $p$ and $p^{r}$, respectively, such that $x_{0}^{p^{r-1}}$ does not commute with $y$.

\section{General case}

Again, let $p$ be a prime, and let $c>1$ be an arbitrary integer. We want to exhibit a capable group $G$ of class exactly $c$, generated by an element of order $p$ and an element of order $p^{1+\lfloor c-1 / p-1\rfloor}$.

Our construction in this section will be based on the nilpotent product of groups; we specialise the definition to the case we are interested in:

Definition 3.1: Let $A_{1}, \ldots, A_{n}$ be cyclic groups, and let $c>0$. The $c$-nilpotent product of the $A_{i}$, denoted $A_{1} \amalg^{\mathfrak{\Re}_{c}} \ldots \amalg^{\mathfrak{\Re}_{c}} A_{n}$ is defined to be the group $F / F_{c+1}$, where $F$ is the free product of the $A_{i}, F=A_{1} * \cdots * A_{n}$, and $F_{c+1}$ is the $(c+1)$-st term of the lower central series of $F$. 
It is easy to verify that the $c$-nilpotent product of the $A_{i}$ is of class exactly $c$, and that it is their coproduct (in the sense of category theory) in the variety $\mathfrak{N}_{c}$ of all nilpotent groups of class at most $c$. The 1-nilpotent product is simply the direct sum of the $A_{i}$.

Note that if $G$ is the $c$-nilpotent product of the $A_{i}$, then $G / G_{k+1}$ is the $k$-nilpotent product of the $A_{i}$ for all $k, 1 \leqslant k \leqslant c$.

We consider $\mathcal{G}=C_{p} \amalg^{\mathfrak{n}_{c+1}} \mathbf{Z}$, the $(c+1)$-nilpotent product of a cyclic group of order $p$ and the infinite cyclic group. Denote the generator of the finite cyclic group by $a$, and the generator of the infinite cyclic group by $z$. Let $G=\mathcal{G} / Z(\mathcal{G})$. Then $G$ is capable of class $c$. We want to show that $z Z(\mathcal{G})$ has the required order.

PROPOSITION 3.2. Let a generate $C_{p}$ and $z$ generate the infinite cyclic group $\mathbf{Z}$. If $\mathcal{G}=C_{p} \amalg^{\mathfrak{\Upsilon}_{c+1}} \mathbf{Z}$, then

$$
Z(\mathcal{G}) \cap\langle z\rangle=\left\langle z^{\left.p^{1+|c-1 / p-1|}\right\rangle .}\right.
$$

Proof: The fact that $z^{p^{1+\lfloor c-1 / p-1\rfloor}}$ is central follows from [5, Theorem 3.16], so we just need to prove the other inclusion. We proceed by induction on $c$. The claim is true if $c=1$ since the commutator bracket is bilinear in a group of class two. Assume the inclusion holds for $c-1$, with $c>1$. Note that $\langle z\rangle \cap \mathcal{G}_{2}=\{e\}$.

Consider $\mathcal{G} / \mathcal{G}_{c+1}$; this is the $c$-nilpotent product of $C_{p}$ and $\mathbf{Z}$, so by the induction hypothesis, the intersection of the centre and the subgroup generated by $z$ is generated by the $p^{1+\lfloor c-2 / p-1\rfloor}$-st power of $z$. Since the center of $\mathcal{G}$ is contained in the pullback of the centre of $\mathcal{G} / \mathcal{G}_{c+1}$, we deduce that the smallest power of $z$ that could possibly be in $Z(\mathcal{G})$ is the $p^{1+\lfloor c-2 / p-1\rfloor}-$ st power.

If $\lfloor c-2 / p-1\rfloor=\lfloor c-1 / p-1\rfloor$, then we are done. So the only case that needs to be dealt with is the case considered in the previous section, when $c=1+(r-1)(p-1)$ for some positive integer $r>1$.

Here we use the universal property of the coproduct. Let $K$ be the group from Lemma 2.2. Since $\mathcal{G}$ is the coproduct of $C_{p}$ and $\mathbf{Z}$ in $\mathfrak{N}_{c+1}$, the morphisms $C_{p} \rightarrow K$ given by $a \mapsto y$, and $\mathbf{Z} \rightarrow K$ given by $z \mapsto x_{0} \in K$, induce a unique homomorphism $\varphi: \mathcal{G} \rightarrow K$. The image of $Z(\mathcal{G})$ must lie in $Z(K)$ (since the map is surjective). Since $\varphi\left(z^{p^{r-1}}\right)=x_{0}^{p^{r-1}}$ does not commute with $y$, we conclude that $z^{p^{r-1}} \notin Z(\mathcal{G})$. This proves that the smallest power of $z$ that could lie in $Z(\mathcal{G})$ is $z^{p^{r}}$, which gives the desired inclusion.

Now let $G=\mathcal{G} / Z(\mathcal{G})$. This is a group of class $c$, minimally generated by $a Z(\mathcal{G})$ and $z Z(\mathcal{G})$. The former has order $p$, and the latter element has order $p^{1+\lfloor c-1 / p-1\rfloor}$ by the proposition above. Thus $G$ is a capable group of class $c$, minimally generated by two elements whose orders satisfy the equality in (1.1), showing that the inequality is indeed best possible.

REMARK 3.3. I believe that in general inequality (1.1) will be both necessary and sufficient for the capability of a $c$-nilpotent product of cyclic $p$-groups. This is indeed the case when $c<p$ and when $p=c=2$; see [5]. However, I have not been able to establish 
this for arbitrary $p$ and $c$, which forced the somewhat indirect approach taken in this note.

\section{REFERENCES}

[1] T.E. Easterfield, 'The orders of products and commutators in prime-power groups', Proc. Cambridge Philos. Soc. 36 (1940), 14-26.

[2] P. Hall, 'The classification of prime-power groups', J. Reine Angew. Math. 182 (1940), 130-141.

[3] H. Heineken and D. Nikolova, 'Class two nilpotent capable groups', Bull. Austral. Math. Soc. 54 (1996), 347-352.

[4] I.M. Isaacs, 'Derived subgroups and centers of capable groups', Proc. Amer. Math. Soc. 129 (2001), 2853-2859.

[5] A. Magidin, 'Capability of nilpotent products of cyclic groups', (preprint, arXiv:math.GR/0403188.

Deptartment of Mathematical Sciences

The University of Montana

Missoula MT 59812

United States of America

e-mail: magidin@member.ams.org 